\title{
Poly-L-lysine-coated nanoparticles are ineffective in inducing mucosal immunity against group a streptococcus
}

\author{
Nirmal Marasini' ${ }^{1}$, Ashwini Kumar Giddam', Michael R. Batzloff², Michael F. Good², Mariusz Skwarczynski ${ }^{1}$ and Istvan Toth ${ }^{1,3,4^{*}}$
}

*Correspondence: i.toth@uq.edu.au

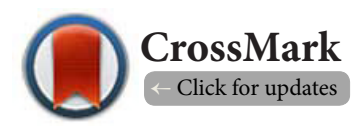

'The University of Queensland, School of Chemistry \& Molecular Biosciences, St Lucia, QLD, 4072, Australia.

${ }^{2}$ Griffith University, Institute for Glycomics, Gold Coast, QLD, 4222, Australia.

${ }^{3}$ The University of Queensland, Institute for Molecular Biosciences, St Lucia, QLD, 4072, Australia.

${ }^{4}$ The University of Queensland, School of Pharmacy, Woolloongabba, QLD, 4102, Australia.

\begin{abstract}
Background: Group A Streptococcus (GAS) can cause a range of maladies, from simple throat infections to lethal complication, such as rheumatic heart disease. The M-protein, a bacterial cell surface protein, is the major virulence factor ofGAS. Several attempts have been made over the past few decades to develop vaccines against GASthat employed peptides derived from the M-protein. One suchapproach used lipopeptides or lipid core peptide (LCP) systems that incorporated a B-cell epitope derived from the conserved region of the M-protein.

Methods: In the present study, we prepared different biodegradable polymer [dextran, poly-(lacticcoglycolic-acid) (PLGA), and poly-L-lysine] nanoparticles (NPs)-based delivery systems for a lipopeptidevaccine candidate (LCP-1).The NPs were characterised by their size, charge, morphology, antigen-presenting cells (APCs) uptake and subsequent APCs maturations efficacy, followed by in vivo nasal immunisation in mice.

Results: All produced NPs ranged in size from 100-205 nm, and their charge varied depending upon the nature of polymer. A high APCs uptake efficacy for dextran and poly-L-lysine NPswere observed, compared to PLGA NPs. Despite the high uptake by APCs, dextran and poly-L-lysine NPs failed to improve APCs maturation that resulted in low antibody titres. In contrast, while LCP-1 encapsulated into PLGA showed low APCs uptake,it induced significant maturation of DCs and higher antibody titres compared to other NPs.
\end{abstract}

Conclusions: Positively-charged poly-L-lysine NPswere non-immunogenic, while negatively charged PLGA NPs induced similar responses to antigens adjuvanted with cholera toxin B (CTB).

Keywords: Mucosal delivery, lipopeptides, nanoparticles, nasal, vaccine, PLGA, Poly-L-lysine

\section{Introduction}

Group A Streptococcus (GAS) infections represent a major health concern indeveloping countries due to poor access to health care facilities. Infections withGAS can lead to minor illnesses, such as pharyngitis, to complicated diseases, such as rhematic heart disease (RHD) [1]. If untreated, GAS infections could be life-threatening; however, antibiotic therapy, particularly penicillin, can be effective in treating the bacterium. Long medication duration can lead to low patient compliance, and chances of re-infection after the completion of therapy are significant problems in disease management [2]. Therefore, the development of a vaccinecould be a promising approach to prevent diseases associated with GAS. GAS invades the body through nasal or respiratory mucosal tissues and then carries out systemic infection. An ideal GAS vaccine would provide both systemic and local protection at the invading sites. Traditional immunisation routes (parenteral routes) may induce systemic immunity, but fail to induce mucosal-specific local protection against administered antigens [3]. In contrast, mucosal immunisation routes, such as nasal, oral, pulmonary, vaginal, etc., are able to induce both mucosal and systemic responses against administered antigens [4]. Due to its antiphagocytic function, the GAS cell-surface M-protein is a major virulence factor inGAS infections. However, the use of whole 
Marasini et al. Biochemical Compounds 2017,

proteins as a vaccine component may be associated with several disadvantages, such as the presence of impurities, unwanted side effects, allergies and manufacturing difficulties [5]. Importantly, some fragments of M-proteins could induce an autoimmune response. Hence, the whole protein is not favoured as an antigen for vaccine development [6]. Therefore, current GAS vaccine development is mainly focused on M-protein-derived peptide-based approaches. However, peptides, themselves, are non-immunogenic and require a delivery system or adjuvant to activate the immune system [6].

Nanoparticles (NPs) based on polymers are a promising platform to enhance the efficacy of mucosally-administered peptide antigens [3]. Cationic NPs have been shown to be effective in inducing mucosal immunity, while anionic NPs have only shown minimum improvement over soluble antigens [7]. We have previously shown that lipopeptides loaded into cationic liposomes or chitosan-based nanoparticles improved mucosal and systemic antibody titres in mice, compared to free lipopeptides, upon intranasal administration [8-10]. PolyL-lysine is a well-known cationic polymer used for the delivery of DNA vaccines [11]. Other polymers, such as dextran and PLGA, were also reportedfor the intranasal delivery of lipopeptide vaccines $[10,12]$. However, intranasal immunisation with lipopeptide antigen loaded poly-L-lysine NPs is yet to be documented. Thus, we expected that NPs coated with poly-Llysine would be preferentially taken up by antigen-presenting cells (APCs), and would generate a strong humoral response upon intranasal delivery. Different NPs incorporating PLGA, dextran and poly-L-lysine were prepared and characterised based on their size, charge and encapsulation efficiency of antigens. Additionally, we measured the capacity of these NPs to be taken up by APCs, and their subsequent effect in the maturation of APCs. Finally, mice were intranasally immunised with the NPs to compare the differences in their capacity to induce both mucosal and systemic immune responses.

\section{Materials and methods}

Poly-(lactic-co-glycolic-acid) (PLGA) (L:G, 50:50) (MW: 10,000$15,000)$ was purchased from PolySciTech ${ }^{\oplus}$ (United States). Dextran sodium (MW: 9,000-20,000), dichloromethane (DCM), poly(vinyl alcohol) (MW: 30,000-70,000), poly-L-lysine hydrobromide (MW: 15,000-30,000), phenylmethylsulfonylfluoride (PMSF), soybean trypsin inhibitor, 3,3'5,5'-Tetramethylbenzidine (TMB) substrate, and all other reagents were purchased at the available purity from Sigma-Aldrich (Castle Hill, NSW, Australia). Trimethyl chitosan (degree of quaternisations: 73\%) was synthesised from chitosan, as described previously [13]. Antimouse $\lg G, \lg G 1$, IgG2a and $\lg A$ antibodies conjugated to horseradish peroxidase were purchased from Sigma-Aldrich (Castle Hill, NSW, Australia). All chemicals were used as received, without any purification. Millipore water was used in all formulations. Lipid core peptide vaccine candidate (LCP-1) (Figure 1) incorporating $C-16$ alkyl lipids (2-amino-D,L-hexadecanoic acid) chemically conjugated by a linker (Ser-Ser-Lys) to B-cell epitope (J14; KQAEDKVKASREAKKQVEKALEQLEDKVK) and universal T-helper cell epitope (P25; KLIPNASLIENCTKAEL) were synthesised as previously described, using the microwave assisted solid phase peptide synthesis method and Boc chemistry $[6,14]$. Additionally, peptides (J14 and P25) were also synthesised by solid phase peptide synthesis using Fmoc chemistry [15].

\section{Preparation of nanoparticles}

The schematic representation for the preparation of NPs is shown in Figure 2. NP-1 was prepared by mixing cationic LCP-1 with anionic dextran in water. NP-2 was prepared by encapsulating NP-1 into PLGA using the double emulsion solvent evaporation method, as reported previously [16]. Briefly, aqueous NPs-1 solution was slowly added to PLGA solution in dichloromethane to prepare water-in-oil (w/o) emulsions. These emulsions were further sonicated and slowly added to the stabiliser solution $(0.75 \%$ PVA in water) to prepare a water-in-oil-in-water (w/o/w) emulsion. Organic phase (dichloromethane) was evaporated by stirring the double emulsion for $6 \mathrm{~h}$ at room temperature. After evaporation, NPs-1-loaded PLGA particles (NPs-2) were subjected to centrifugation at $3,000 \mathrm{~g}$ for $3 \mathrm{~min}$. The sediment and pellets were discarded,

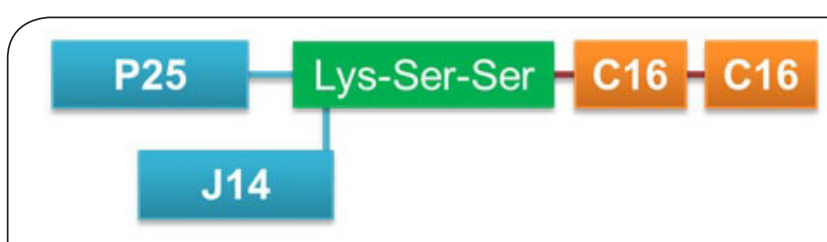

J14: KQAEDKVKASREAKKQVEKALEQLEDKVK P25: KLIPNASLIENCTKAEL<smiles>CC(C)(C)CCCCC(N)C(=O)O</smiles>

Figure 1. Structure of LCP-1.

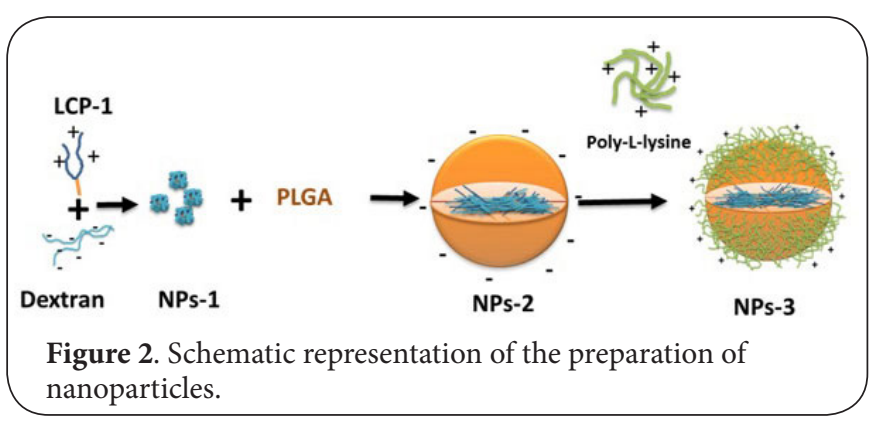


and the supernatant was further centrifuged at $15,000 \mathrm{~g}$ for $15 \mathrm{~min}$ to form pellets. The final supernatant was discarded and pellets were washed twice through dispersion in Millipore water, followed by centrifugation at $15,000 \mathrm{~g}$ for $15 \mathrm{~min}$. The final pellets were redispersed in water. NPs-3 were prepared as above, except aqueous poly-L-lysine solution was added to NP-2 after evaporating dichloromethane, and stirred for a further $2 \mathrm{~h}$ at room temperature.

\section{Particle size distribution and zetapotentials}

The particle size, polydispersity index and zeta potential of the NPs were characterised by dynamic light scattering (DLS) using a Zetasizer (Zetasizer Nano Series ZS, Malvern Instruments, United Kingdom). Analyses of all the NPs were performed at least in triplicate.

\section{Morphology}

Transmission electron microscopy (TEM, JEOL Ltd, Japan) was used to visualise the surface morphology of the NPs. NPs-1 was visualised without any stain, while visualisation of NPs2 and NPs-3 was done with $1 \%$ phosphotungstic acid stain. Briefly, a drop of NPs was settled into carbon-coated copper grid for $1 \mathrm{~min}$ and excess was wicked off using filter paper. A drop of staining solution was added and left for 30 s. Excess stain solution was removed using filter paper. The grid was air-dried for $5 \mathrm{~min}$ before observing them by microscope.

Antigen-presenting cells uptake and maturation studies Spleens were harvested from naïve mice and processed as described previously without any modification [13]. The splenocytes cells were stimulated with NPs containing carboxyfluroscein conjugated to LCP-1 $(2 \mu \mathrm{g})$ for APCs uptake studies $[10,12]$. In the maturation studies, a similar amount of LCP-1 (without carboxyfluroscein) containing NPs was used. The uptake of LCP-1 and the expression of costimulatory maturation markers by APCs were quantified by flow cytometry (LSR II Flowcytometer, BD Biosciences, CA, USA).

\section{Immunisation studies}

Swiss out-bred mice were used for the nasal immunisation study. The animal ethics and experimental protocols were carefully reviewed and approved by the Institute of Ethics Review Board (Griffith University, GU ref no.Gly-01-15 AEC)for animal-based work, in accordance with National Health and Medical Research Council (NHMRC) of Australia guidelines. Mice were divided into different groups ( $\mathrm{n}=5 \mathrm{mice} / \mathrm{group}$ ) and immunised with NPs bearing $10 \mu \mathrm{g}$ of LCP-1. Mice were administered intranasally with a booster dose after 2 weeks post-primary immunisation. Positive control mice were administered with $10 \mu \mathrm{g}$ cholera toxin B (CTB) and $20 \mu \mathrm{g}$ of peptides (J14 and P25). The negative control group was administered with PBS. All of the formulations were administered at a volume of $10 \mu \mathrm{L}(5 \mu \mathrm{L} /$ nare). Samples (saliva) were collected after the $7^{\text {th }}$ day of first boosts upon intraperitoneal injection with pilocarpine solution $(50 \mu \mathrm{L})$. Blood sample were collected on $14^{\text {th }}$ day of post-primary immunization. Sera was separated after centrifuging blood at $10,000 \mathrm{~g}$ for $10 \mathrm{~min}$. Both samples were stored at $-80^{\circ} \mathrm{C}$ before final analysis. ELISA was performed on all samples to determine the production of J-14-specific antibodies by the previously reported method [8].

\section{Statistical analysis}

One-way analysis of variance (ANOVA) and Tukey post-hoc tests were used for statistical analyses. $P$-values of $<0.05(*)$ were considered statistically significant.

\section{Results and discussion}

Vaccination is considered to be one of the greatest human weapons in the fight against a wide range of infectious diseases [17]. Gram-positive GAS bacteria invade humans through the respiratory mucosa, and could cause fatal complication, such as rheumatic heart disease. The M-protein is the major virulence factor for GAS infection. One of the directions for vaccine development is the use of conserved $B$-cell epitopes from the M-protein (e.g. J14) $[5,18,19]$. However, peptide-based vaccines are not immunogenic and require the addition of adjuvants or a delivery system for optimum efficacy. Recently, the J14 B-cell peptide epitope, along with the P25 universal T-helper epitope, were introduced into the lipid core peptide (LCP) system to form vaccine candidates against GAS (Figure 1) $[20,21]$. This lipopeptide, when delivered with the cationic liposome, chitosan, and PLGA-based nanocarriers significantly improved immune responses over free LCP-1 upon mucosal immunizations $[\mathbf{8}, \mathbf{9}, \mathbf{1 3}, \mathbf{1 5}, \mathbf{1 6}]$. However, it is not clear if high immune responses are due to NPs materialor its cationic charge. Therefore, we hypothesised that cationic poly-ı-lysine bearing NPs would potentially improve the antigen-specific immune response of LCP-1 after intranasal immunisation.

Negatively charged NPs were prepared either by complexing LCP-1 with dextran (NPs-1) or encapsulating NPs-1 into PLGA (NPs-2). NPs-2 were further coated with poly-L-lysine to form positively charged NPs-3. Dextran, PLGA and poly-L-lysine were selected due to their biodegradability, low toxicity and adjuvanting properties [22]. Particle size plays a vital role in the ability to cross mucosal membranes and be taken up by APCs [19]. Therefore, all particles were produced in the size range of 100-205 nm. Specifically, DLS analysis showed that the diameter of NPs-1, NPs-2 and NPs-3 were $129 \pm 0.2 \mathrm{~nm}$, $193 \pm 4 \mathrm{~nm}$ and $205 \pm 1 \mathrm{~nm}$, respectively, and the polydispersity index were $0.21 \pm 0.01,0.08 \pm 0.04$, and $0.11 \pm 0.04$, respectively, indicating a monodispersed size distribution of particles. TEM analysis of NPs confirmed these particle sizes (Figure 3). The surface-charge of NPs- $1, N P s-2$ and NPs-3 were $-50 \pm 0.5$ $\mathrm{mV},-31 \pm 0.5 \mathrm{mV}$, and $+15 \pm 1.0 \mathrm{mV}$, respectively.

APCs, such as dendritic cells (DCs) or macrophages, take up, process and present antigens to naïve T-cells through the major histocompatibility complex, which stimulates antibody response against specific antigens. To determine whether NPs 


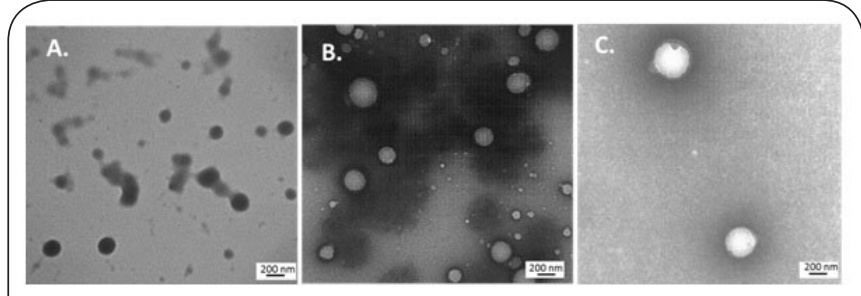

Figure 3. TEM images of A) NPs-1; B) NPs-2; and C) NPs-3. NPs-1 were visualised without stain, while NPs-2 and NPs-3 were stained with $1 \%$ phosphotungstic acid stains.

were able to be taken up by APCs, NPs were incubated with splenocyte-derived macrophages and dendritic cells (Figure 4). Dextran/LCP-1 bearing anionic NPs (NPs-1) and cationic polyL-lysine coated PLGA NPs (NPs-3) were taken up by both DCs and macrophages significantly better than free LCP-1. NPs-1 were taken up by APCs preferentially, as expected, due to the well-documented recognition of dextran by mannose receptors present in the APCs [23]. Similarly, cationic NPs-3 were promptly taken up by DCs and macrophages. However, the uptake of LCP-1 was significantly reduced when lipopeptide was incorporated into PLGA-based polymers (NPs-2).

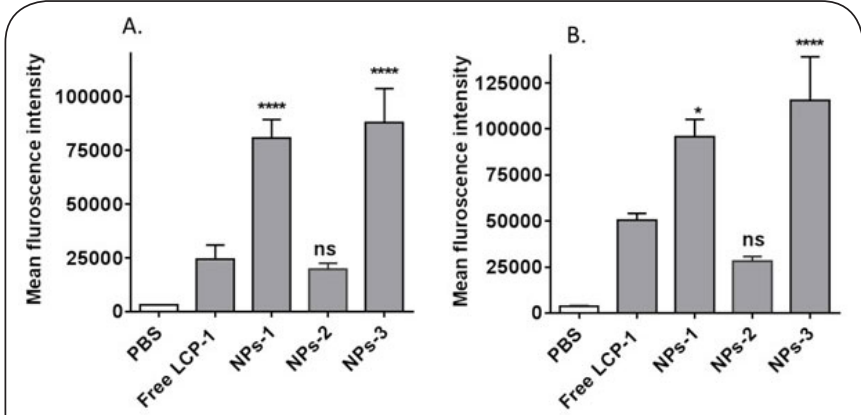

Figure 4. The uptake of different nanoparticles by dendritic cells (A) and macrophages (B) compared with free LCP$1(n=3)$.Statistical significances amongst the groups were compared using ANOVA and Tukey post-hoc testing. ${ }^{*} p<0.05$.

The ability of the NPs to induce maturation of APCs were accessed. When APCs mature, they express different co-stimulatory molecules, such as CD40, CD80 and CD86. In general, LCP-1 and NPs-1 did not significantly increase the expression of markers at the tested lipopeptide concentration(Figure 5). Despite its low uptake, NPs-2 were able to induce a signifi-

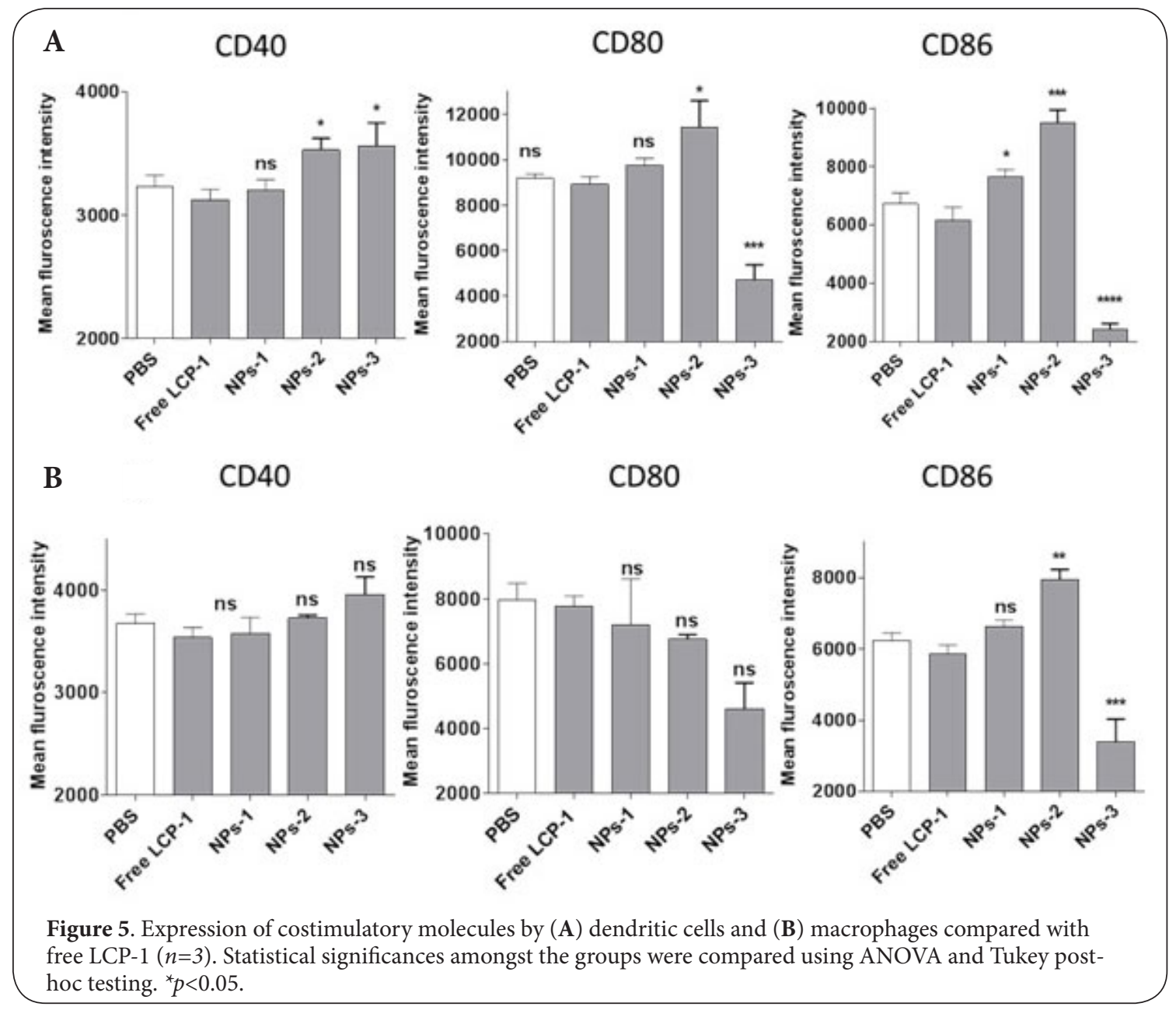


cantly higher expression of costimulatory molecules in DCs. Interestingly, in spite of its high uptake, poly-L-lysine bearing NPs down-regulated the expression of CD80 and CD86 in DCs. The potential ability of poly-L-lysine to down-regulate an immune response had been suggestedpreviously [24]. In contrast, the expression of CD40 was significantly enhanced by NPs-3. Similar trends in the expression of co-stimulatory molecules in macrophages was observed for all studied groups. Thus, NPs-3 might not be able to stimulate maturation of APCs and induce humoral immunity.

The production of antigen-specific mucosal and systemic antibody titres were examined following intranasal immunisation with NPs in Swiss outbred mice. A physical mixture of CTB with peptides (J14 and P25) was used as a positive control, while PBS was used as negative control. Following two immunisations, free LCP-1 and dextran complexed LCP-1 (NP-1) did not induce significant levels of systemic lgG titres (Figure 6A). In contrast, antibody titres were markedly improved when J14 peptide epitopes were administered with CTB. Interestingly, the incorporation of anionic dextran/LCP-1 into PLGA polymers (NPs-2) showed significant improvement in antibody titres, while additional cationic poly-L-lysine coating (NPs-3) did not induce production of the significant systemic antibody titres. These results were consistent with APCs maturation experiments where NPs-2 showed a high expression of co-stimulatory markers, while NPs-3 showed down-regulation of co-stimulatory markers. High levels of J14-specific systemic antibody titres are expected to reduce systemic dissemination of GAS, while the reduction of mucosal colonisation requires a high level of IgA antibodies in mucosal tissues. Except for the CTB-adjuvanted group, where two out of five mice produced J14-specific IgA titres, none other groups showed an induction of mucosal immunity (Figure 6B). Taken together, we observed that mice immunised with a low dose of LCP-1-bearing PLGA NPs generated a humoral immune response against the antigen, while the poly-L-lysine polymer did not provide an adjuvanting effect. This could be related

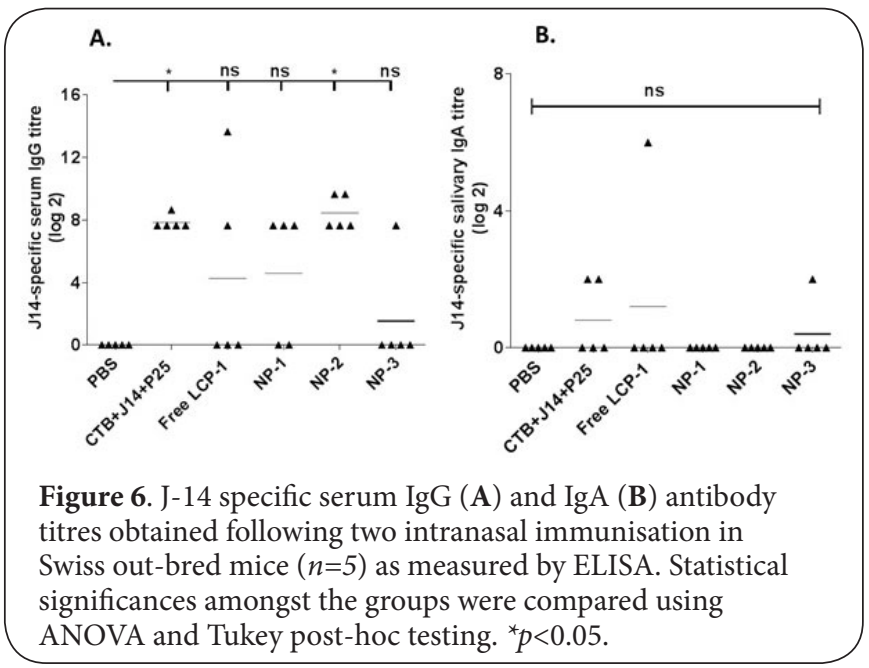

to the polymers capacity to downregulate APCs maturation

\section{Conclusions}

In conclusion, we developed NPs-based delivery systems for lipopeptide-based vaccines. Although there was low uptake of PLGA-bearing NPs by APCs, they proved to be the most efficient in the maturation of APCs and the induction of systemic lgG antibodies compared to free lipopeptides or negative controls. In contrast to our expectations, although highly taken up by APCs, cationic poly-L-lysine bearing nanoparticles were unable to trigger in vitro maturation and antibody responses. Thus, nanoparticle charge is not the crucial factor for induction of humoral immune response in mucosal immunization.

\section{Competing interests}

The authors declare that they have no competing interests.

Authors' contributions
\begin{tabular}{|l|c|c|c|c|c|c|}
\hline Authors' contributions & NM & AKG & MRB & MFG & MS & IT \\
\hline Research concept and design & $\checkmark$ & -- & -- & -- & $\checkmark$ & $\checkmark$ \\
\hline Collection and/or assembly of data & $\checkmark$ & $\checkmark$ & $\checkmark$ & $\checkmark$ & -- & -- \\
\hline Data analysis and interpretation & $\checkmark$ & -- & -- & -- & $\checkmark$ & -- \\
\hline Writing the article & $\checkmark$ & -- & -- & -- & -- & -- \\
\hline Critical revision of the article & $\checkmark$ & -- & -- & -- & $\checkmark$ & $\checkmark$ \\
\hline Final approval of article & $\checkmark$ & $\checkmark$ & $\checkmark$ & $\checkmark$ & $\checkmark$ & $\checkmark$ \\
\hline Statistical analysis & $\checkmark$ & -- & -- & -- & -- & -- \\
\hline
\end{tabular}

\section{Acknowledgement and funding}

The authors acknowledge the scientific and technical resources of the Australian Microscopy \& Microanalysis Research Facility at the Centre for Microscopy and Microanalysis, The University of Queensland. NirmalMarasini is a recipient of an International Postgraduate Research Scholarship (IPRS) and Australian Postgraduate Award (APA) for his PhD study. This work was supported by the National Health and Medical Research Council [NHMRC Program Grant 496600].

\section{Publication history}

Editor: Yingying Xu, North Dakota State University, USA.

Received: 07-Nov-2016 Final Revised: 06-Jan-2017

Accepted: 10-Feb-2017 Published: 28-Feb-2017

\section{References}

1. Wong SS and Yuen KY. Streptococcus pyogenes and re-emergence of scarlet fever as a public health problem. Emerg Microbes Infect. 2012; 1:e2. | Article | PubMed Abstract | PubMed FullText

2. Baldassarri L, Creti R, Recchia S, Imperi M, Facinelli B, Giovanetti $E$, Pataracchia M, Alfarone $G$ and Orefici G. Therapeutic failures of antibiotics used to treat macrolide-susceptible Streptococcus pyogenes infections may be due to biofilm formation. J Clin Microbiol. 2006; 44:2721-7. | Article | PubMed Abstract | PubMed FullText

3. Marasini N, Skwarczynski M and Toth I. Oral delivery of nanoparticlebased vaccines. Expert Rev Vaccines. 2014; 13:1361-76. | Article | PubMed

4. Lycke N. Recent progress in mucosal vaccine development: potential and limitations. Nat Rev Immunol. 2012; 12:592-605. | Article | PubMed

5. Skwarczynski $M$ and Toth I. Recent advances in peptide-based subunit nanovaccines. Nanomedicine (Lond). 2014; 9:2657-69. | Article | PubMed 
6. Skwarczynski M and Toth I. Peptide-based subunit nanovaccines. Curr Drug Deliv. 2011; 8:282-9. | Article | PubMed

7. Tada R, Hidaka A, Iwase N, Takahashi S, Yamakita Y, Iwata T, Muto S, Sato E, Takayama N, Honjo E, Kiyono H, Kunisawa J and Aramaki Y. Intranasal Immunization with DOTAP Cationic Liposomes Combined with DCCholesterol Induces Potent Antigen-Specific Mucosal and Systemic Immune Responses in Mice. PLoS One. 2015; 10:e0139785. | Article | PubMed Abstract I PubMed FullText

8. Ghaffar KA, Marasini N, Giddam AK, Batzloff MR, Good MF, Skwarczynski $M$ and Toth I. Liposome-based intranasal delivery of lipopeptide vaccine candidates against group A streptococcus. Acta Biomater. 2016; 41:1618. | Article | PubMed

9. Marasini N, Ghaffar KA, Giddam AK, Batzloff MR, Good MF, Skwarczynski $M$ and Toth I. Highly Immunogenic Trimethyl Chitosan-Based Delivery System for Intranasal Lipopeptide Vaccines against Group A Streptococcus. Curr Drug Deliv. 2016. | Article | PubMed

10. Marasini N, Giddam AK, Khalil ZG, Hussein WM, Capon RJ, Batzloff MR, Good MF, Toth I and Skwarczynski M. Double adjuvanting strategy for peptide-based vaccines: trimethyl chitosan nanoparticles for lipopeptide delivery. Nanomedicine (Lond). 2016; 11:3223-3235. | Article | PubMed

11. Minigo G, Scholzen A, Tang CK, Hanley JC, Kalkanidis M, Pietersz GA, Apostolopoulos $V$ and Plebanski M. Poly-L-lysine-coated nanoparticles: a potent delivery system to enhance DNA vaccine efficacy. Vaccine. 2007; 25:1316-27. | Article | PubMed

12. Marasini N, Khalil ZG, Giddam AK, Ghaffar KA, Hussein WM, Capon RJ, Batzloff MR, Good MF, Skwarczynski M and Toth I. Lipid core peptide/ poly(lactic-co-glycolic acid) as a highly potent intranasal vaccine delivery system against Group A streptococcus. Int J Pharm. 2016; 513:410-420. | Article | PubMed

13. Marasini N, Giddam AK, Ghaffar KA, Batzloff MR, Good MF, Skwarczynski $M$ and Toth I. Multilayer engineered nanoliposomes as a novel tool for oral delivery of lipopeptide-based vaccines against group A Streptococcus. Nanomedicine (Lond). 2016; 11:1223-36. | Article | PubMed

14. Abdel-Aal AB, Batzloff MR, Fujita Y, Barozzi N, Faria A, Simerska P, Moyle PM, Good MF and Toth I. Structure-activity relationship of a series of synthetic lipopeptide self-adjuvanting group a streptococcal vaccine candidates. J Med Chem. 2008; 51:167-72. | Article | PubMed

15. Chan A, Hussein WM, Ghaffar KA, Marasini N, Mostafa A, Eskandari S, Batzloff MR, Good MF, Skwarczynski M and Toth I. Structure-activity relationship of lipid core peptide-based Group A Streptococcus vaccine candidates. Bioorg Med Chem. 2016; 24:3095-101. | Article | PubMed

16. Marasini N, Khalil ZG, Giddam AK, Ghaffar KA, Hussein WM, Capon RJ, Batzloff MR, Good MF, Skwarczynski M and Toth I. Lipid core peptide/ poly(lactic-co-glycolic acid) as a highly potent intranasal vaccine delivery system against Group A streptococcus. Int J Pharm. 2016; 513:410-420. | Article | PubMed

17. Chandrudu S, Skwarczynski M, Pattinson D, Apte SH, Doolan DL and Toth I. Synthesis and immunological evaluation of peptide-based vaccine candidates against malaria. Biochemical Compounds. 2016; 4:1. | Article

18. Skwarczynski $M$ and Toth I. Lipid-core-peptide system for selfadjuvanting synthetic vaccine delivery. Methods Mol Biol. 2011; 751:297-308. | Article | PubMed

19. Skwarczynski M and Toth I. Peptide-based synthetic vaccines. Chemical Science. 2016; 7:842-54.

20. Zaman M, Abdel-Aal AB, Fujita Y, Phillipps KS, Batzloff MR, Good MF and Toth I. Immunological evaluation of lipopeptide group A streptococcus (GAS) vaccine: structure-activity relationship. PLoS One. 2012; 7:e30146. | Article | PubMed Abstract | PubMed FullText

21. Zaman M, Abdel-Aal AB, Fujita Y, Ziora ZM, Batzloff MR, Good MF and Toth I. Structure-activity relationship for the development of a selfadjuvanting mucosally active lipopeptide vaccine against Streptococcus pyogenes. J Med Chem. 2012; 55:8515-23. | Article | PubMed

22. Wang $S$, Liu $H$, Zhang $X$ and Qian F. Intranasal and oral vaccination with protein-based antigens: advantages, challenges and formulation strategies. Protein Cell. 2015; 6:480-503. | Article | PubMed Abstract | PubMed FullText

23. Dasgupta S, Navarrete AM, Bayry J, Delignat S, Wootla B, Andre S, Christophe O, Nascimbeni M, Jacquemin M, Martinez-Pomares L, Geijtenbeek TB, Moris A, Saint-Remy JM, Kazatchkine MD, Kaveri SV and Lacroix-Desmazes $S$. A role for exposed mannosylations in presentation of human therapeutic self-proteins to $\mathrm{CD} 4+\mathrm{T}$ lymphocytes. Proc Natl Acad Sci U S A. 2007; 104:8965-70. | Article | PubMed Abstract | PubMed FullText

24. Phillips B, Nylander K, Harnaha J, Machen J, Lakomy R, Styche A, Gillis K, Brown L, Lafreniere D, Gallo M, Knox J, Hogeland K, Trucco M and Giannoukakis N. A microsphere-based vaccine prevents and reverses new-onset autoimmune diabetes. Diabetes. 2008; 57:1544-55. | Article | PubMed Abstract | PubMed FullText

\section{Citation:}

Marasini N, Giddam AK, Batzloff MR, Good MF, Skwarczynski M and Toth I. Poly-L-lysine-coated nanoparticles are ineffective in inducing mucosal immunity against group a streptococcus. Bio Chem Comp. 2017; 5:1.

http://dx.doi.org/10.7243/2052-9341-5-1 